\title{
Celastrol attenuates oxidative stress in the skeletal muscle of diabetic rats by regulating the AMPK-PGC1 $\alpha-S I R T 3$ signaling pathway
}

\author{
YUE GUAN $^{1}$, ZI-JIAN CUI ${ }^{2}$, BEI SUN ${ }^{1}$, LI-PING HAN ${ }^{1}$, CHUN-JUN LI $^{1}$ and LI-MING CHEN ${ }^{1}$ \\ ${ }^{1}$ Key Laboratory of Hormones and Development (Ministry of Health), Tianjin Key Laboratory of Metabolic Diseases, \\ Tianjin Metabolic Diseases Hospital and Tianjin Institute of Endocrinology, Tianjin Medical University, Tianjin 300070; \\ ${ }^{2}$ Department of Spinal Surgery, Tianjin Union Medicine Center, Tianjin 300121, P.R. China
}

Received December 8, 2015; Accepted March 28, 2016

DOI: $10.3892 /$ ijmm.2016.2549

\begin{abstract}
Oxidative stress plays a key role in the pathogenesis of diabetic myopathy. Celastrol provides a wide range of health benefits, including antioxidant, anti-inflammatory and antitumor effects. We hypothesized that celastrol may exert an antioxidant effect in the skeletal muscle of diabetic rats. In the present study, MnSOD activity was determined by spectrophotometry. The protein levels were evaluated by western blot analysis and mRNA content was quantified by RT-qPCR. We firstly found that the levels of AMP-activated protein kinase (AMPK), peroxisome proliferator-activated receptor coactivator $1 \alpha(\mathrm{PGC} 1 \alpha)$, silent mating-type information regulation 2 homolog 3 (SIRT3) and manganese superoxide dismutase (MnSOD) were all decreased in the skeletal muscle of diabetic patients. Male rats with diabetes were also treated with the vehicle or with celastrol at 1 , 3 and $6 \mathrm{mg} / \mathrm{kg} /$ day for 8 weeks. The administration of celastrol at 3 and $6 \mathrm{mg} / \mathrm{kg}$ attenuated the deterioration of skeletal muscle, as shown by histological analysis, decreased the malondialde-
\end{abstract}

Correspondence to: Dr Li-Ming Chen or Dr Chun-Jun Li, Key Laboratory of Hormones and Development (Ministry of Health), Tianjin Key Laboratory of Metabolic Diseases, Tianjin Metabolic Diseases Hospital and Tianjin Institute of Endocrinology, Tianjin Medical University, 66 Tongan Road, Heping, Tianjin, Hebei 300070, P.R. China

E-mail: drlimingchen@sina.com

E-mail: 1i_chunjun@126.com

Abbreviations: T2DM, type 2 diabetes mellitus; ROS, reactive oxygen species; MnSOD, manganese superoxide dismutase; AMPK, AMP-activated protein kinase; PGC1 $\alpha$, peroxisome proliferatoractivated response- $\gamma$ coactivator $1 \alpha$; Sirt3, silent mating-type information regulation 2 homolog 3; GSH, glutathione; MDA, malondialdehyde; SOD, superoxide dismutase; FPG, fasting plasma glucose; BUN, blood urea nitrogen; AST, aspartate aminotransferase; ALT, alanine aminotransferase; TC, total cholesterol; TG, triglyceride; TBS, Tris-buffered saline; HbAlc, glycated haemoglobin A1c; HDL-C, high-density lipoprotein cholesterol, LDL-C, low-density lipoprotein cholesterol; $\mathrm{SCr}$, serum creatinine

Key words: diabetes, oxidative stress, celastrol, silent mating-type information regulation 2 homolog 3 , manganese superoxide dismutase hyde (MDA) level and increased the glutathione (GSH) level assayed by enzyme-linked immunosorbent assay (ELISA) method. It also enhanced the enzyme activity and increased the expression of MnSOD, and increased the AMPK phosphorylation level, as well as PGCl $\alpha$ and Sirt3 expression. The findings of our study suggest that the expression of AMPK, PGC1 $\alpha$, SIRT3 and MnSOD are decreased in the skeletal muscle of diabetic patients. Celastrol exerted antioxidant effects on skeletal muscle partly by regulating the AMPK-PGC1 $\alpha$-SIRT3 signaling pathway.

\section{Introduction}

Diabetic myopathy, characterized by a reduction in muscle mass, strength and physical capacity $(1,2)$, is a serious, but often overlooked complication of diabetes that contributes to an overall worsening of the diabetic condition. Studies have reported that the skeletal muscle of individuals with type 2 diabetes mellitus (T2DM) exhibits an increased number of glycolytic fibers (3), muscle atrophy (4) and a decrease in capillary density (5). Functional impairments are also evident, as demonstrated by a decline in muscle strength and motor dysfunction (6). The pathogenesis of diabetic myopathy is very complex.

Oxidative stress, induced by an abundance of reactive oxygen species (ROS) or by the failure of the antioxidant defense machinery, is considered a critical factor for the pathogenesis of diabetes (7). Manganese superoxide dismutase (MnSOD), also known as SOD2, is a primary mitochondrial antioxidant that neutralizes mitochondrial ROS through the conversion of superoxide to hydrogen peroxide, and ultimately to $\mathrm{H}_{2} \mathrm{O}$ by catalase (8). In humans, mutations in MnSOD are associated with age-related disorders, such as cardiovascular disease, insulin resistance and diabetes, indicating that upstream signaling proteins that regulate MnSOD may also play a role in these pathologies (9).

AMP-activated protein kinase (AMPK) is an ubiquitous heterotrimeric serine/threonine protein kinase, which functions as a fuel sensor in a number of tissues, including skeletal muscle (10). The activation of AMPK enhances the mRNA expression of peroxisome proliferator-activated response- $\gamma$ coactivator-1 $\alpha$ (PGC1 $\alpha$ ) and MnSOD (11). PGC1 $\alpha$, as a pivotal factor for mitochondrial function, has been shown to be essential for the regulation of mitochondrial metabolism, biogenesis and oxidative stress (12). 
As a member of the sirtuin family, silent mating-type information regulation 2 homolog 3 (SIRT3) is mainly localized in the mitochondria and regulates several pivotal oxidative pathways by targeting several enzymes involved in central metabolism (13). It has been demonstrated that SIRT3 functions as a downstream target of $\mathrm{PGC} 1 \alpha$, which is directly regulated by AMPK and has multiple cellular functions by deacetylating mitochondrial proteins, as well as MnSOD (14-16). Thus, based on the data of previous studies $(17,18)$ indicating that the AMPKPGC1 $\alpha$-SIRT3 pathway and MnSOD modulates mitochondrial biogenesis and oxidative stress, we hypothesized that the levels of AMPK, PGC1 $\alpha$, SIRT3 and MnSOD may be all decreased in the skeletal muscle of individuals with diabetes, which may be responsible for increased oxidative stress. Our findings demonstrated that the AMPK-PGC1 $\alpha$-SIRT3 signaling pathway was downregulated in the skeletal muscle of diabetic patients.

Celastrol is a triterpenoid compound extracted from the Chinese herb, Tripterygium wilfordii Hook.f., which is known to have exert various biological effects, including immunosuppressive, anti-inflammatory and antitumor effects (19). Nowadays, it is widely used in the treatment of diabetic nephropathy (20). Celastrol has been proven to exert antioxidant effects, and has been shown to reduce ROS generation in hypertensive rats and vascular smooth muscle cells (21). It also can promote the glutathione (GSH) redox cycle by increasing the intracellular GSH content and the GSH/GSSG ratio in macrophages (19). However, the antioxidant effect of celastrol on skeletal muscle in individuals with diabetes has not been fully investigated. Thus, in this study, we examined the effects of celastrol on oxidative stress in the skeletal muscle of diabetic rats, as well as the potential involvement of the AMPK-PGC1 $\alpha$-SIRT3 signaling pathway. Our findings demonstrated that celastrol exerted antioxidant effects in the skeletal muscle of diatetic rats, and that these effects were partly mediated by the activation of the AMPK-PGC1 $\alpha$-SIRT3 signaling pathway.

\section{Materials and methods}

Patients and sample collection. Approval for this study was obtained from the Ethics Committee of the Metabolic Disease Hospital of Tianjin Medical University, Tianjin, China and informed consent was obtained from all participants prior to orthopedic surgery. This trial has been verified by the Chinese Clinical Trial Registry, and its registration number is ChiCTR-COC-15007025. All participants were patients with lumbar disc herniation who required orthopedic surgery. Among these patients, 10 patients suffered from T2DM and the another 10 were non-diabetic patients. The patients with diabetes had been previously diagnosed at the hospital. Patients with cardiovascular disease, neoplastic disease, neurodegenerative disease and acute inflammation were excluded from the study.

Blood samples were collected from the antecubital vein at the first day after admission. All blood was drawn from the patients at the same time in the morning (between 6 and 8 a.m.). Laboratory test results were evaluated, including fasting plasma glucose (FPG), blood urea nitrogen (BUN), creatinine, aspartate aminotransferase (AST), alanine aminotransferase (ALT) and glycated haemoglobin A1c (HbA1c), levels, and lipid profiles, including total cholesterol (TC), high-density lipoprotein cholesterol (HDL-C), low-density lipoprotein cholesterol (LDL-C) and triglyceride (TG) levels. All the laboratory parameters were assessed using conventional laboratory methods. The blood samples were centrifuged for $15 \mathrm{~min}$ at 3,000 rpm (AU5400; Olympus, Tokyo, Japan), and plasma was assayed for the laboratory parameters using an automated analyzer (7600A-020; Hitachi, Tokyo, Japan)

Between 300 and $500 \mathrm{mg}$ of paravertebral skeletal muscle was obtained from patients undergoing lumbar vertebral disc decompression discectomy. Dissections of skeletal muscle were obtained within $10 \mathrm{~min}$ of delivery and snap-frozen in liquid nitrogen and stored at $-80^{\circ} \mathrm{C}$ until further analysis. Tissues were also embedded in paraffin for histological analysis by hemotoxylin and eosin (H\&E) staining. For all surgerical procedures, a spinal anesthesia and/or epidural were used. All muscle samples were obtained at the time of surgery (between 8:30 a.m. and 13:00 p.m.). All patients were fasted overnight.

Animals and experimental design. Male Sprague-Dawley (SD) rats ( $\mathrm{n}=90,6$ weeks old), weighing $161 \pm 9 \mathrm{~g}$, were purchased from Beijing Hua Fu Kang Biotechnology Co., Ltd. (Beijing, China). Rats were given free access to food and tap water and were caged individually under a 12-h light-dark cycle at a temperature of $22 \pm 3^{\circ} \mathrm{C}$ and humidity of $55 \pm 5 \%$. All animal experiments were conducted in accordance with the Principles of Laboratory Care, and approved by the Institutional Animal Care and Use Committee, Metabolic Disease Hospital of Tianjin Medical University.

The rats were randomly divided into the control (NC) and the high energy diet (HED) groups. In the control group, the animals received a standard chow diet, while the rats in the HED group were fed with an additional high energy emulsion, as previously described $(22,23)$. After 8 weeks on their respective diets, streptozotocin (STZ; $45 \mathrm{mg} / \mathrm{kg}$; Sigma, St. Louis, MO, USA) dissolved in $0.1 \mathrm{~mol} / 1$ citrate buffer ( $\mathrm{pH} 4.5)$ was injected into the caudal vein of the rats in the HED group to establish a model of T2DM, while the rats in the control group were injected with sodium citrate buffer. The rats with blood glucose levels $\geq 16.7 \mathrm{mmol} / \mathrm{l}$ at 7 days after the STZ injection were selected as the model of diabetes. On average, $80 \%$ of the rats injected with STZ met these criteria. At 1 week following the injection of STZ, the rats with successfullyinduced diabetes were randomly divided into the diabetes model (DM) group, the celastrol low-dose group (1 mg/kg/day), the celastrol middle-dose group ( $3 \mathrm{mg} / \mathrm{kg} / \mathrm{day})$ and the celastrol high-dose group ( $6 \mathrm{mg} / \mathrm{kg} / \mathrm{day})(\mathrm{n}=15$ rats per group). The rats in the treatment groups were administered celastrol by gavage, whereas the rats in the NC and DM groups were administered an equal amount of distilled water $(2 \mathrm{ml})$. Following 8 weeks of the respective treatments, rats were anesthetized with an intraperitoneal injection of sodium pentobarbital $(30 \mathrm{mg} / \mathrm{kg}$ body weight) and tissue samples were collected for analysis. The paravertebral muscle was excised from the rat bodies, and was cut perpendicularly along the longitudinal axis and fixed in phosphate-buffered $20 \%$ formaldehyde. Histological paraffinembedded sections $(5 \mu \mathrm{m})$ were then prepared for H\&E staining. The sections of paravertebral muscle were snap-frozen in liquid nitrogen and stored at $-80^{\circ} \mathrm{C}$ until further analysis.

Measurement of biochemical and physical parameters. Body weight and fasting blood glucose (FBG) levels were measured 
each week. The overnight-fasted rats were weighed by electronic balance (AM1100; Mettler-Toledo AG, Schwerzenbach, Switzerland) at the same time in the morning (between 6 and 8 a.m.). Then blood samples were obtained from the caudal vein and FBG levels were measured using a Accu-Chek Aviva glucometer (Roche Diagnostics, Mannheim, Germany). After 8 weeks of treatment, the rats were euthanized. Blood samples were obtained from the retroorbital venous plexus by using plain microhematocrit capillary tubes tubes (VWR, West Chester, PA, USA) and collected into tubes containing EDTA at the time of sacrifice and were centrifuged at $3,000 \mathrm{x} \mathrm{g} / \mathrm{min}$ for $15 \mathrm{~min}$. Plasma was separated for measuring FPG, BUN, serum creatinine (SCr), AST, ALT, TC and TG using an automatic biochemistry analyzer (CD-1600CS; Abbott Labs, North Chicago, IL, USA).

Measurements of mitochondrial oxidative biochemical parameters. Mitochondrial-enriched supernatants were prepared from frozen skeletal muscle samples as previously described (24). The content of malondialdehyde (MDA) and GSH was measured by enzyme-linked immunosorbent assay (ELISA) using respective kits following the manufacturer's instructions (Jiancheng Co. Ltd., Nanjing, China). MnSOD activity was determined by spectrophotometry. Briefly, reaction buffer (50 mM sodium phosphate, $0.1 \mathrm{mM}$ EDTA, $0.01 \mathrm{mM}$ xanthine, and $0.01 \mathrm{mM}$ cytochrome $c, \mathrm{pH} 7.8$ ) was mixed with $0.005 \mathrm{U} / \mathrm{ml}$ xanthine oxidase and $2 \mathrm{mM}$ sodium cyanide, and the absorbance change at $550 \mathrm{~nm}$ was followed. Then sample was added stepwise (in $20 \mu \mathrm{l}$ increments at a concentration of $5-10 \mathrm{mg} / \mathrm{ml}$ ), and the concentration of sample required to decrease the rate of reaction by $50 \%$ (defined as 1 unit of MnSOD activity) was calculated. Enzyme values are presented as units per milligram of protein.

SIRT3 activity assays. Mitochondrial-enriched supernatants were prepared from frozen skeletal muscle samples as described (24). SIRT3 enzyme activity in the gastrocnemius mitochondria was assayed using a fluorometric kit (Biomoles, Inc., Shoreline, WA, USA) as per the manufacturer's instructions. Briefly, $25 \mu \mathrm{l}$ distilled water, $5 \mu \mathrm{l}$ buffer, $5 \mu \mathrm{l}$ fluoro-substrate peptide $5 \mu \mathrm{l} \mathrm{NAD}$ and $5 \mu \mathrm{l}$ developer were added to each microtiter plate wells and mixed well. Reactions were initiated by adding $5 \mu \mathrm{l}$ samples or buffer of samples or recombinant SIRT3 to matching well and mixing thoroughly. SIRT3 activity was measured using a fluorimetric microplate reader at $450 \mathrm{~nm}$ with an excitation of $350 \mathrm{~nm}$. We measured and calculated the rate of reaction, while the reaction velocity remained constant. The enzyme activity was normalized to the total protein content of each sample and the results are expressed relative to the mean for the NC group.

Western blot analysis. Protein lysates were obtained by homogenizing paravertebral muscle with lysis buffer. The protein concentration was measured using the Bio-Rad protein assay (Bio-Rad, Richmond, CA, USA). Approximately $50 \mu \mathrm{g}$ protein was subjected to $10 \%$ sodium dodecyl sulfate-polyacrylamide gel electrophoresis (SDS-PAGE) and transferred onto nitrocellulose membranes. After blocking with 5\% dried milk in Tris-buffered saline (TBS) containing $0.1 \%$ Tween-20 for $2 \mathrm{~h}$, the membranes were subsequently incubated overnight with primary antibodies diluted in 5\% dried milk-TBS containing $0.1 \%$ Tween-20.
The primary antibodies were as follows: anti-AMPK (\#2532, 1:1,000), anti-phosphorylated (p-)AMPK (:\#2531, 1:1,000) (both from Cell Signaling Technology, Danvers, MA, USA), anti- $\alpha$ tubulin (sc-8035, 1;10,000), anti- $\beta$-actin (sc-47778, 1:10,000) (both from Santa Cruz Biotechnology, Inc., Santa Cruz, CA, USA), anti-SIRT3 (ab86671, 1:500), anti-MnSOD (ab13534, 1:2,000) and anti-PGC1 $\alpha$ (ab54481, 1:1,000) (all from Abcam Cambridge, MA, USA) antibodies. The membranes were then incubated with appropriate horseradish-peroxidase-conjugated secondary antibodies (sc-2005 and sc-2003, Santa Cruz Biotechnology, Inc.). An enhanced chemiluminescence system was used to visualize the bands. Densitometric analysis was performed using a gel image analysis system (UVP Inc., San Gabriel, CA, USA).

Reverse transcription-quantitative PCR (RT-qPCR). The MnSOD, Sirt3 and PGC1 $\alpha$ mRNA levels were quantified by SYBR-Green Real-Time PCR (Invitrogen, Carlsbad, CA, USA). RT-qPCR was performed as previously described (25). RNA was extracted from paravertebral muscle using TRIzol reagent (Invitrogen). Reverse transcription was performed using the RevertAid First Strand cDNA Synthesis kit (Thermo Fisher Scientific, Inc., Waltham, MA, USA). The quantitative PCR (qPCR) measurement of individual cDNAs was carried out using SYBR-Green dye to measure duplex DNA formation with the LightCycler System (Roche Diagnostics). The data were analyzed using Bio-Rad CFX manager software 1.6. The quantified results were normalized to those of GADPH, using the 2-( $\Delta \Delta \mathrm{CT})$ method. The nucleotide sequences of the PCR primers used were as follows: MnSOD forward, 5'-ACTGAAGTTCAATGGTGGGG-3' and reverse, 5'-GCTTGATAGCCTCCAGCAAC-3'; Sirt3 forward, 5'-TAC AGAAATCAGTGCCCCGA-3' and reverse, 5'-GGTGGAC ACAAGAACTGCTG-3'; PGC1 $\alpha$ forward, 5'-ATGAGAA GCGGGAGTCTGAA-3' and reverse, 5'-GCGGTCTCTCA GTTCTGTCC-3'; GAPDH forward, 5'-TGCCACTCAG AAGACTGTGG-3' and reverse, 5'-TTCAGCTCTGGGATG ACCTT-3'.

Statistical analysis. All data are presented as the means \pm SD. Differences between groups were analyzed using the Student's t-test and one-way analysis of variance (ANOVA) test using the statistical software. SPSS 18.0 (SPSS Inc., Chicago, IL, USA). Values of $\mathrm{p}<0.05$ were considered to indicate statistically significant differences.

\section{Results}

Comparison between diabetic and non-diabetic patients Comparison of physical and biochemical parameters between diabetic and non-diabetic patients. There were no significant differences in gender, age and body weight between the normal control and diabetic patients. The FPG, HbA1c, TG, TC and LDL-C levels were significantly higher in the diabetic patients compared to the normal controls (all $\mathrm{P}<0.01$ ). Although the HDL-C levels were decreased in the diabetic patients, there were no significant differences between the 2 groups (Table I).

Changes in skeletal muscle of diabetic patients. H\&E staining of the paraspinal muscle of non-diabetic patients was presented as normal (Fig. 1A and C), whereas the skeletal 

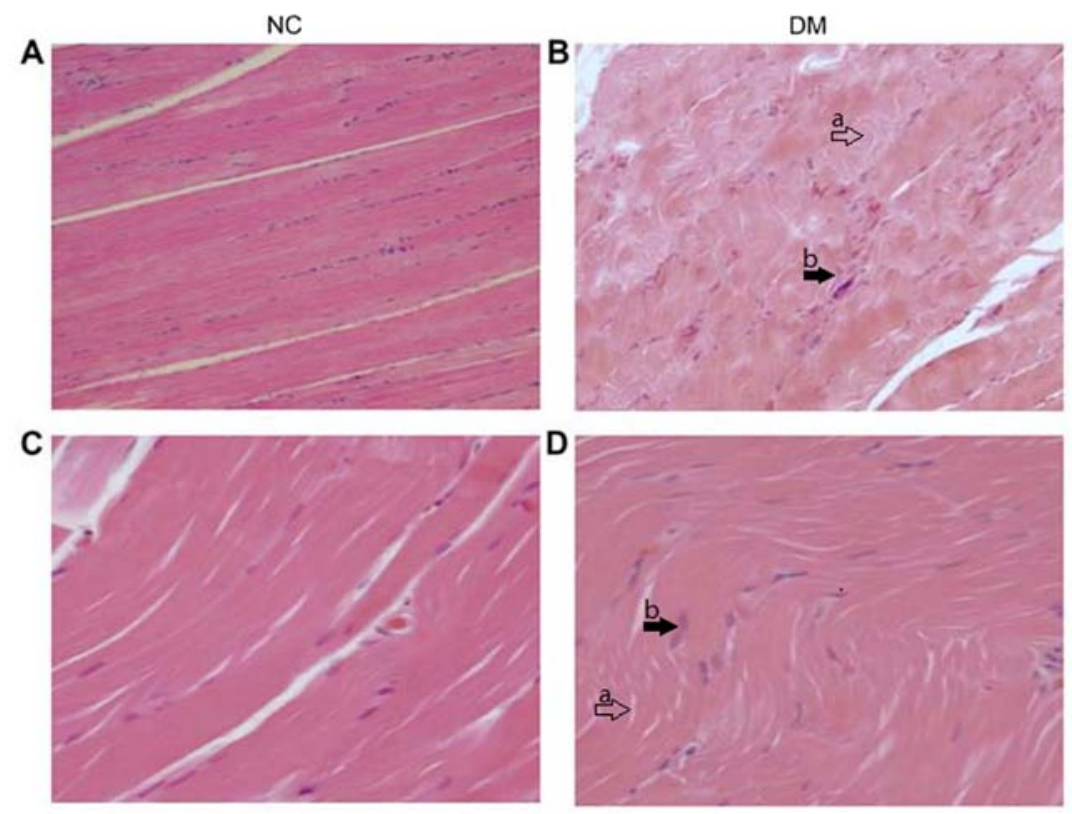

Figure 1. Hemotoxylin and eosin (H\&E) staining of skeletal muscle of diabetic and non-diabetic patients. Images show the longitudinal section of paravertebral muscle. (A and B) Magnification x100; (C and D) magnification x400. Arrow ' $a$ ' shows the irregular fiber structure, with wide gaps; arrow 'b' shows the increased number of nuclei and some with an abnormal position, partly inserted in the muscle fibers.

Table I. Physical and biochemical parameters of diabetic and non-diabetic patients.

\begin{tabular}{lccc}
\hline Parameter & NC & \multicolumn{1}{c}{ DM } & P-value \\
\hline Gender (M/F) & \multicolumn{1}{c}{$7 / 3$} & \multicolumn{1}{c}{$6 / 4$} & $\mathrm{P}=0.64$ \\
Age (years) & $60.80 \pm 6.02$ & $62.10 \pm 5.80$ & $\mathrm{P}=0.65$ \\
BW (kg) & $77.05 \pm 5.37$ & $79.40 \pm 5.84$ & $\mathrm{P}=0.41$ \\
FPG (mmol/l) & $5.13 \pm 0.42$ & $8.70 \pm 1.01$ & $\mathrm{P}<0.01$ \\
HbA1c (\%) & $5.54 \pm 0.24$ & $7.55 \pm 0.57$ & $\mathrm{P}<0.01$ \\
TG (mmol/l) & $1.59 \pm 0.33$ & $2.31 \pm 0.61$ & $\mathrm{P}<0.01$ \\
TC $(\mathrm{mmol} / \mathrm{l})$ & $4.73 \pm 0.36$ & $5.44 \pm 0.35$ & $\mathrm{P}<0.01$ \\
HDL-C (mmol/l) & $1.34 \pm 0.09$ & $1.28 \pm 0.10$ & $\mathrm{P}=0.20$ \\
LDL-C (mmol/l) & $2.58 \pm 0.33$ & $3.47 \pm 0.29$ & $\mathrm{P}<0.01$ \\
\hline
\end{tabular}

Values are expressed as the means \pm standard deviation (SD); $\mathrm{NC}$, normal control (non-diabetic patients); DM, diabetic patients, $\mathrm{n}=10$ per group. BW, body weight; FPG, fasting plasma glucose; HbA1c, glycated haemoglobin A1c; TG, triglyceride; TC, total cholesterol; HDL-C, high-density lipoprotein cholesterol; LDL-C, low-density lipoprotein cholesterol.

muscle of the diabetic patients exhibited an irregular fiber structure, with wide gaps, nuclei of diverse sizes, an increased number of nuclei and some with an abnormal position, partly inserted in the muscle fibers (Fig. 1B and D).

MnSOD enzyme activity is decreased in skeletal muscle of diabetic patients. Both an increase in ROS production and a decline in ROS clearance can lead to increased mitochondrial oxidative stress (26). In the present study, we focused on MnSOD, the primary antioxidative enzyme in mitochondria. Compared to the control group, MnSOD enzyme activity in the diabetes group was decreased by approximately 50\% (Fig. 2A). We also found that the diabetic patients had lower mRNA levels of MnSOD by approximately 50\% (Fig. 2B) and a decreased protein content of MnSOD (Fig. 2C) compared to the non-diabetic control group.

The AMPK-PGCI $\alpha$-SIRT3 pathway is downregulated in the skeletal muscle of diabetic patients. It has been demonstrated that SIRT3 and MnSOD protein abundance is regulated in a signaling axis involving both AMPK and PGCl $\alpha$ (27). Thus, in this study, we compared the expression of the AMPK-PGC1 $\alpha$-SIRT3 signaling pathway between diabetic and non-diabetic patients. As shown in Fig. 3, compared with the non-diabetic patients, the PGCl $\alpha$ and SIRT3 mRNA levels were decreased by almost 63 and $47 \%$, respectively in the diabetic patients (Fig. 3A and B). The protein levels of PGC1 $\alpha$ (Fig. 3D) and SIRT3 (Fig. 3E) were similar to those of the mRNA levels, and were decreased by 60 and $50 \%$ in the diabetic patients, respectively. Western blot analysis revealed an approximate $60 \%$ decrease in AMPK phosphorylation levels in the diabetic patients, which was determined by the p-AMPK levels normalized to the total AMPK levels (Fig. 3C).

Evaluation of the effects of celastrol on the skeletal muscle of diabetic rats. According to the results of our clinical research, we found that the levels of MnSOD were reduced in the skeletal muscle of diabetic patients, and that the AMPK-PGC1 $\alpha$-SIRT3 pathway was downregulated in the skeletal muscle of diabetic patients. In view of the antioxidant effects of celastrol and that it is widely used in the treatment of diabetic nephropathy, we examined the antioxidant effects of this drug in the skeletal muscle of diabetic rats. We preliminarily investigated whether the antioxidant effects of celastrol are mediated through the AMPK-PGC1 $\alpha$-SIRT3 signaling pathway, and compared the effects of different doses of celastrol on this pathway.

Effects of celastrol on biochemical and physical parameters of experimental rats. Table II shows the biochemical results for each group. As expected, the FPG concentrations were signifi- 

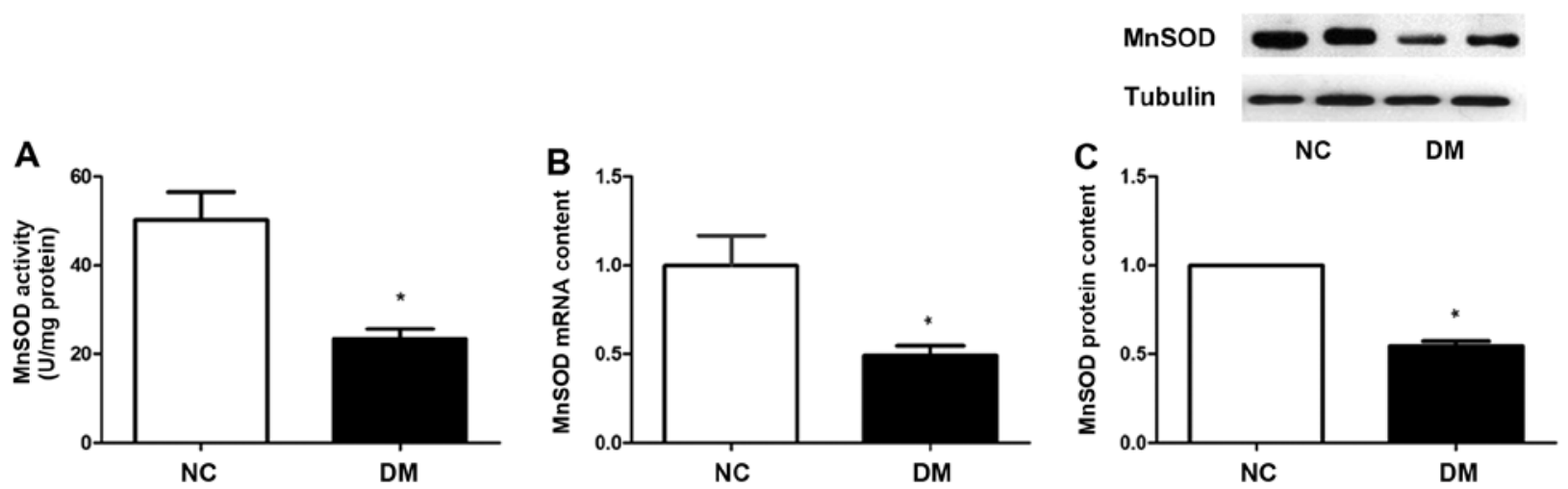

Figure 2. (A) Manganese superoxide dismutase (MnSOD) enzyme activity, and (B) mRNA and (C) protein expression were reduced in the skeletal muscle of diabetic patients compared with the normal controls (NC group). ${ }^{*} \mathrm{p}<0.05$ vs. normal control.
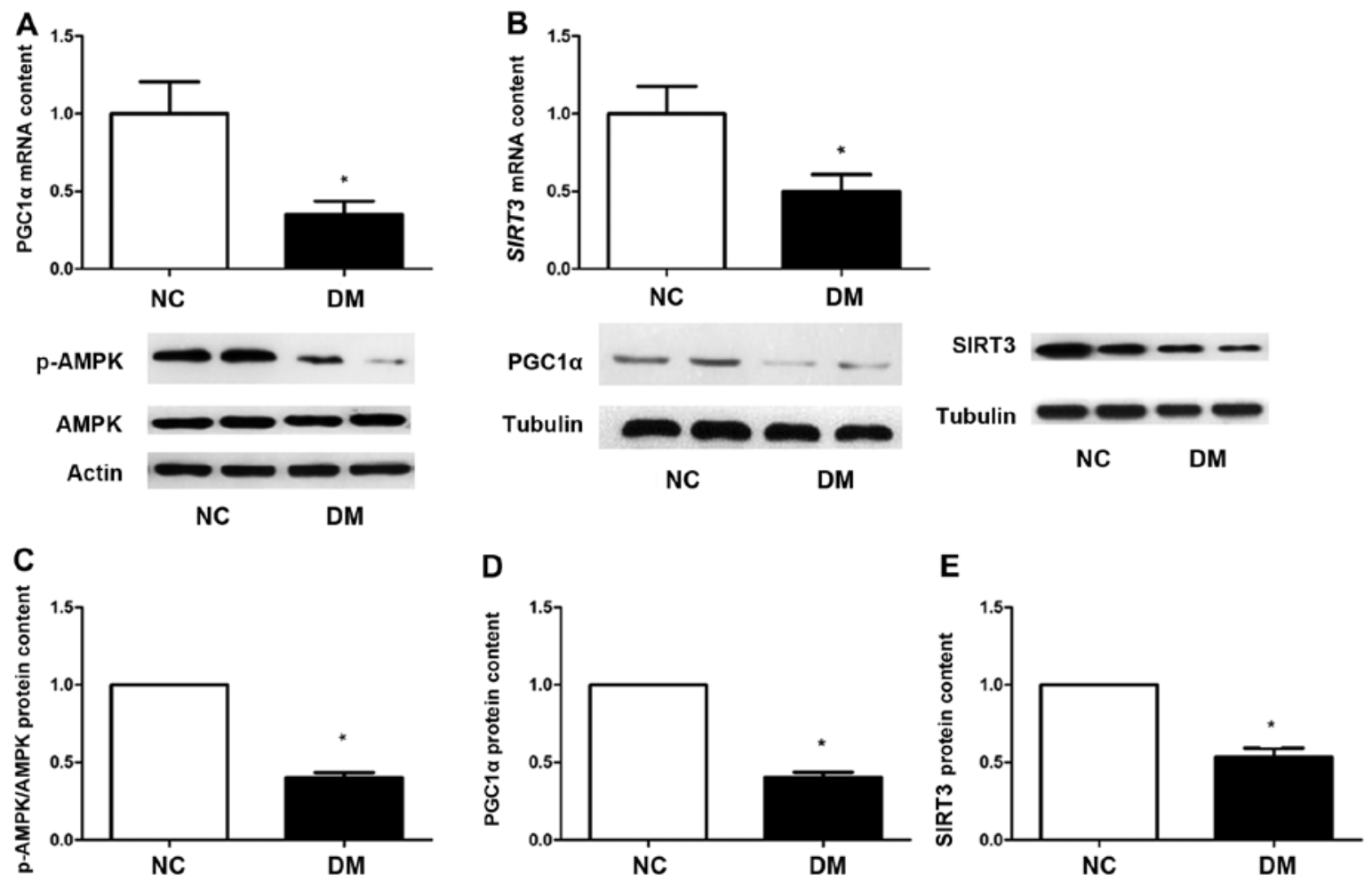

Figure 3. Peroxisome proliferator-activated response- $\gamma$ coactivator $1 \alpha(\mathrm{PGC} 1 \alpha)(\mathrm{A}) \mathrm{mRNA}$ content and (D) protein content were reduced in diabetic patients compared with non-diabetic patients. As a downstream target of PGCl $\alpha$, similar results were observed for (B and E) silent mating-type information regulation 2 homolog 3 (SIRT3). (C) The level of phosphoryled AMP-activated protein kinase (AMPK) (p-AMPK/AMPK) in skeletal muscle was also decreased in diabetic patients compared with non-diabetic patients. " $\mathrm{p}<0.05$ vs. normal control.

cantly higher, while body weight was lower in the diabetic rats compared to the normal control rats throughout the experimental period. However, there were no significant differences in the plasma levels of ALT, AST, BUN and SCr between the normal control rats and the diabetic rats. Of note, the TG and TC content increased significantly in the diabetic rats compared with the normal control rats due to the high fat diet (HED). Although it has been reported that treatment with celastrol significantly decreases body weight, blood glucose levels, and plasma TC and TG levels (28), 8 weeks of treatment with various doses of celastrol had no significant effect on body weight, the FPG level, and plasma TC and TG levels in the present study.

Effects of celastrol on pathological changes in the skeletal muscle of experimental rats. $\mathrm{H} \& \mathrm{E}$ staining of the paraspinal muscle of the rats in the $\mathrm{NC}$ group exhibited a normal morphology (Fig. 4A and F). However, while the skeletal muscle of the rats in the DM group exhibited an irregular fiber structure, with wide gaps, nuclei of diverse sizes, an increased number of nuclei and some with an abnormal position, partly inserted in the muscle fibers (Fig. 4B and G). Following treatment with celastrol, pathological damage was attenuated in varying degrees compared with the rats in the DM group in a dose-dependent manner (Fig. 4C-E and H-J).

Celastrol decreases the levels of markers of oxidative stress. Markers of oxidative stress had been found to be altered in mice with type 2 diabetes and in individuals with diabetes $(29,30)$. Thus, in this study, we analyzed mitochondrial oxidative stress by measuring the levels of MDA, GSH and MnSOD. 
Table II. Effects of celastrol on physical and biochemical parameters of experimental animals.

\begin{tabular}{|c|c|c|c|c|c|c|}
\hline Parameter & Week & $\mathrm{NC}$ & DM & $\begin{array}{c}\mathrm{DM}+\text { celastrol } \\
1 \mathrm{mg} / \mathrm{kg}\end{array}$ & $\begin{array}{c}\mathrm{DM}+\text { celastrol } \\
3 \mathrm{mg} / \mathrm{kg}\end{array}$ & $\begin{array}{c}\mathrm{DM}+\text { celastrol } \\
6 \mathrm{mg} / \mathrm{kg}\end{array}$ \\
\hline \multirow[t]{2}{*}{$\mathrm{BW}(\mathrm{g})$} & 0 & $397.6 \pm 41.8^{a}$ & $309.8 \pm 32.3$ & $320.8 \pm 18.7$ & $333.3 \pm 22.8$ & $321.7 \pm 21.1$ \\
\hline & 8 & $442.7 \pm 43.8^{a}$ & $298.6 \pm 37.1$ & $318.2 \pm 22.1$ & $308.6 \pm 37.8$ & $328.5 \pm 16.8$ \\
\hline \multirow[t]{2}{*}{ FPG (mmol/l) } & 0 & $6.2 \pm 0.2^{\mathrm{a}}$ & $24.2 \pm 8.6$ & $26.7 \pm 2.1$ & $23.9 \pm 4.1$ & $28.8 \pm 2.8$ \\
\hline & 8 & $6.0 \pm 0.2^{\mathrm{a}}$ & $28.2 \pm 3.0$ & $25.8 \pm 6.9$ & $27.6 \pm 5.7$ & $29.0 \pm 3.1$ \\
\hline ALT (U/l) & 8 & $49.7 \pm 9.5$ & $55.1 \pm 5.1$ & $53.8 \pm 10.9$ & $54.9 \pm 9.7$ & $54.2 \pm 8.9$ \\
\hline $\operatorname{AST}(\mathrm{U} / \mathrm{l})$ & 8 & $105.1 \pm 18.4$ & $109.4 \pm 8.4$ & $116.8 \pm 9.5$ & $99.5 \pm 15.9$ & $112.2 \pm 13.9$ \\
\hline BUN (mmol/l) & 8 & $10.9 \pm 2.0$ & $10.7 \pm 1.6$ & $10.9 \pm 1.3$ & $10.0 \pm 1.9$ & $10.2 \pm 1.2$ \\
\hline $\mathrm{SCr}(\mu \mathrm{mol} / \mathrm{l})$ & 8 & $32.8 \pm 4.4$ & $31.3 \pm 3.3$ & $32.8 \pm 3.5$ & $31.1 \pm 4.1$ & $31.5 \pm 3.8$ \\
\hline $\mathrm{TG}(\mathrm{mmol} / \mathrm{l})$ & 8 & $1.5 \pm 1.2^{\mathrm{a}}$ & $4.4 \pm 1.8$ & $4.0 \pm 2.2$ & $3.7 \pm 1.8$ & $3.5 \pm 1.4$ \\
\hline $\mathrm{TC}(\mathrm{mmol} / \mathrm{l})$ & 8 & $2.2 \pm 1.2^{\mathrm{a}}$ & $6.9 \pm 0.9$ & $6.6 \pm 2.1$ & $6.4 \pm 2.2$ & $6.2 \pm 2.0$ \\
\hline
\end{tabular}

Values are expressed as the means \pm standard deviation (SD), ${ }^{a} \mathrm{p}<0.05$ vs. diabetic rats. NC, normal control; DM, diabetic model, $\mathrm{n}=15$ animals per group. BW, body weight; FPG, fasting plasma glucose; ALT, alanine aminotransferase; AST, aspartate aminotransferase; BUN, blood urea nitrogen; SCr, serum creatinine; TG, triglyceride; TC, total cholesterol.
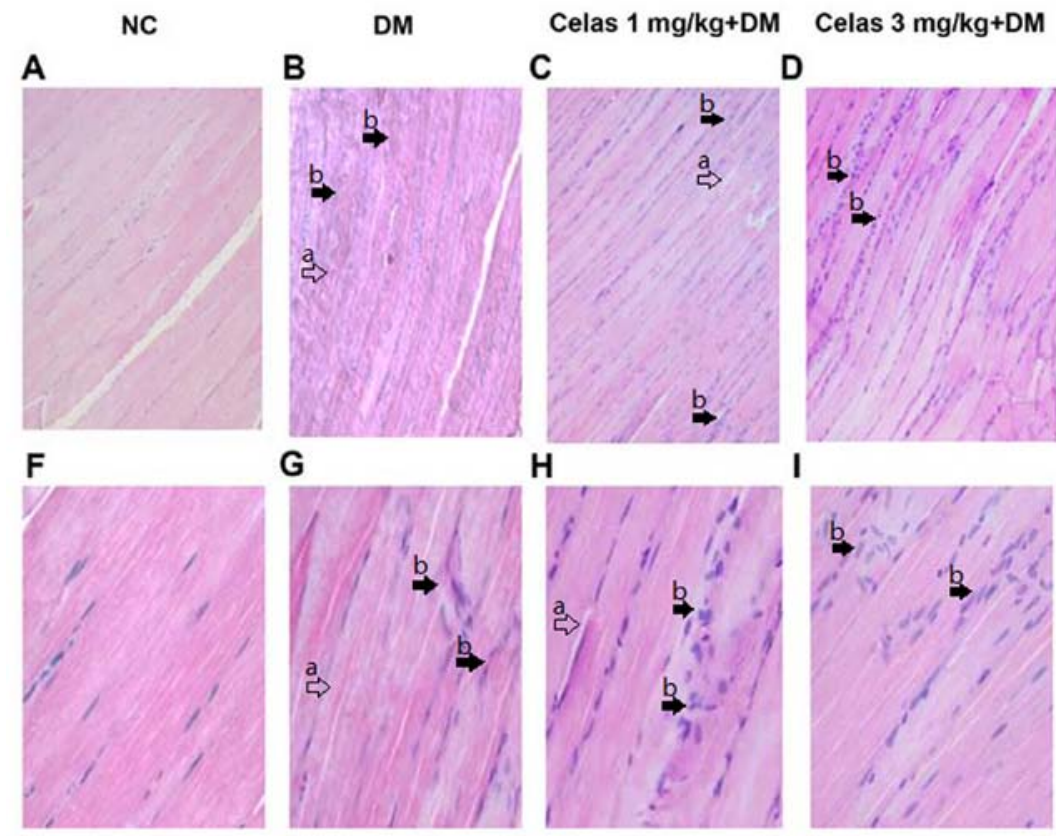

Celas $6 \mathrm{mg} / \mathrm{kg}+\mathrm{DM}$

E

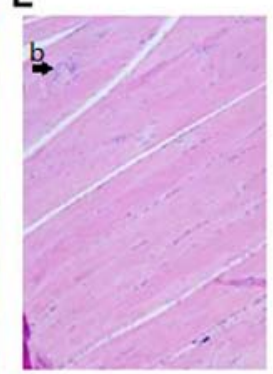

J

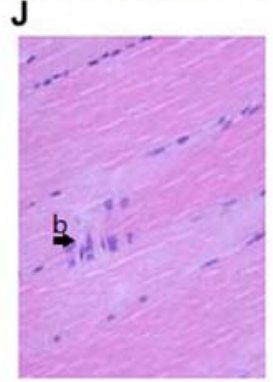

Figure 4. Hemotoxylin and eosin (H\&E) staining of the skeletal muscle of experimental rats. Images show the longitudinal section of the gastrocnemius muscle. (A-E) Magnification, x100; (F-J) magnification, x400. Arrow 'a' shows the irregular fiber structure, with wide gaps; arrow 'b' shows the increased number of nuclei and some with an abnormal position, partly inserted in the muscle fibers.

The level of MDA in the DM group was 2-fold higher than that of the NC group. Following treatment with 3 and $6 \mathrm{mg} / \mathrm{kg}$ celastrol, the levels of MDA were significantly decreased by 35.2 and $36.7 \%(\mathrm{P}<0.05)$, respectively; while there was no significant difference in the levels of MDA between the DM group and the group treated with celastrol $1 \mathrm{mg} / \mathrm{kg}$ (Fig. 5A). By contrast, the level of GSH was decreased by $59.2 \%$ due to the onset of diabetes. Treatment with 3 and $6 \mathrm{mg} / \mathrm{kg}$ celastrol markedly restored the GSH level $(\mathrm{P}<0.05)$ to almost normal levels. However, treatment with $1 \mathrm{mg} / \mathrm{kg}$ celastrol had no significant effect compared with the DM group ( $\mathrm{P}>0.05$; Fig. 5B).
As an antioxidant defense mechanism, increased MnSOD can partly indicate the attenuation of oxidative stress (31). In this study, we found that the diabetic animals had 64.2 and $52.5 \%$ lower mRNA and protein levels of MnSOD, respectively compared to the NC group. Treatment with 3 and $6 \mathrm{mg} /$ $\mathrm{kg}$ celastrol significantly increased these levels. The results of western blot analysis also indicated that the MnSOD protein level was upregulated in the paravertebral muscle of rats treated with 3 and $6 \mathrm{mg} / \mathrm{kg}$ celastrol (Fig. 6A and B). Furthermore, we analyzed the enzyme activity of MnSOD. We also found that treatment with 3 and $6 \mathrm{mg} / \mathrm{kg}$ celastrol markedly enhanced 

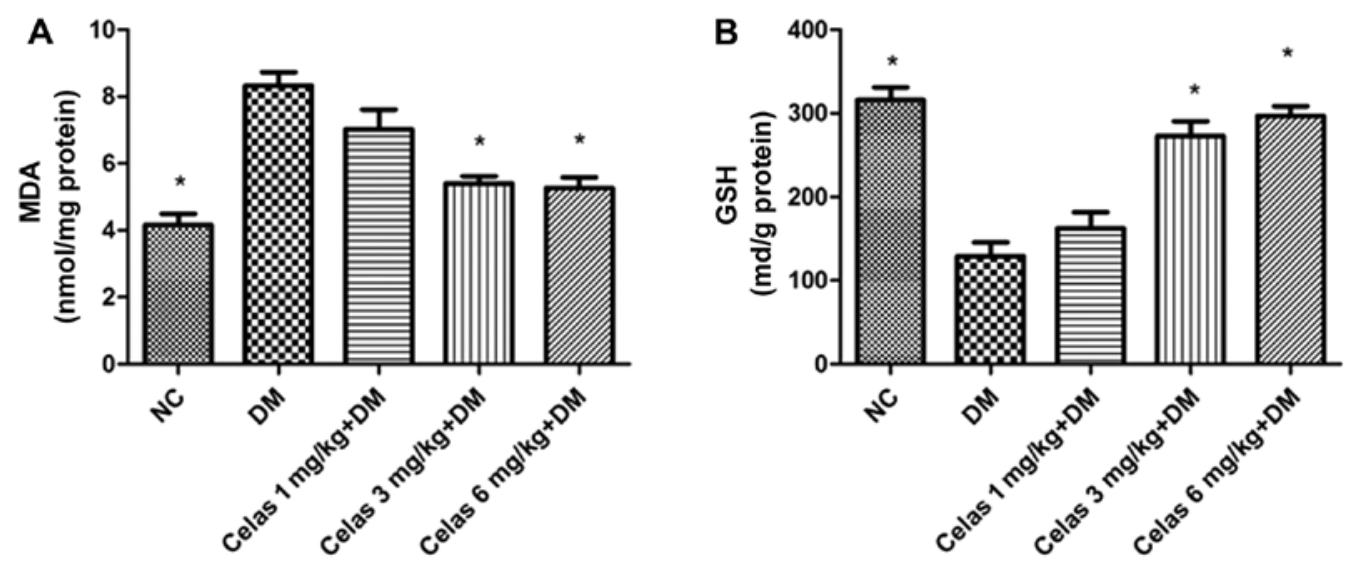

Figure 5. Effect of celastrol treatment on the levels of (A) malondialdehyde (MDA) and (B) glutathione (GSH) in paraspinal muscle of experimental rats p $<0.05$ vs. diabetic rats.
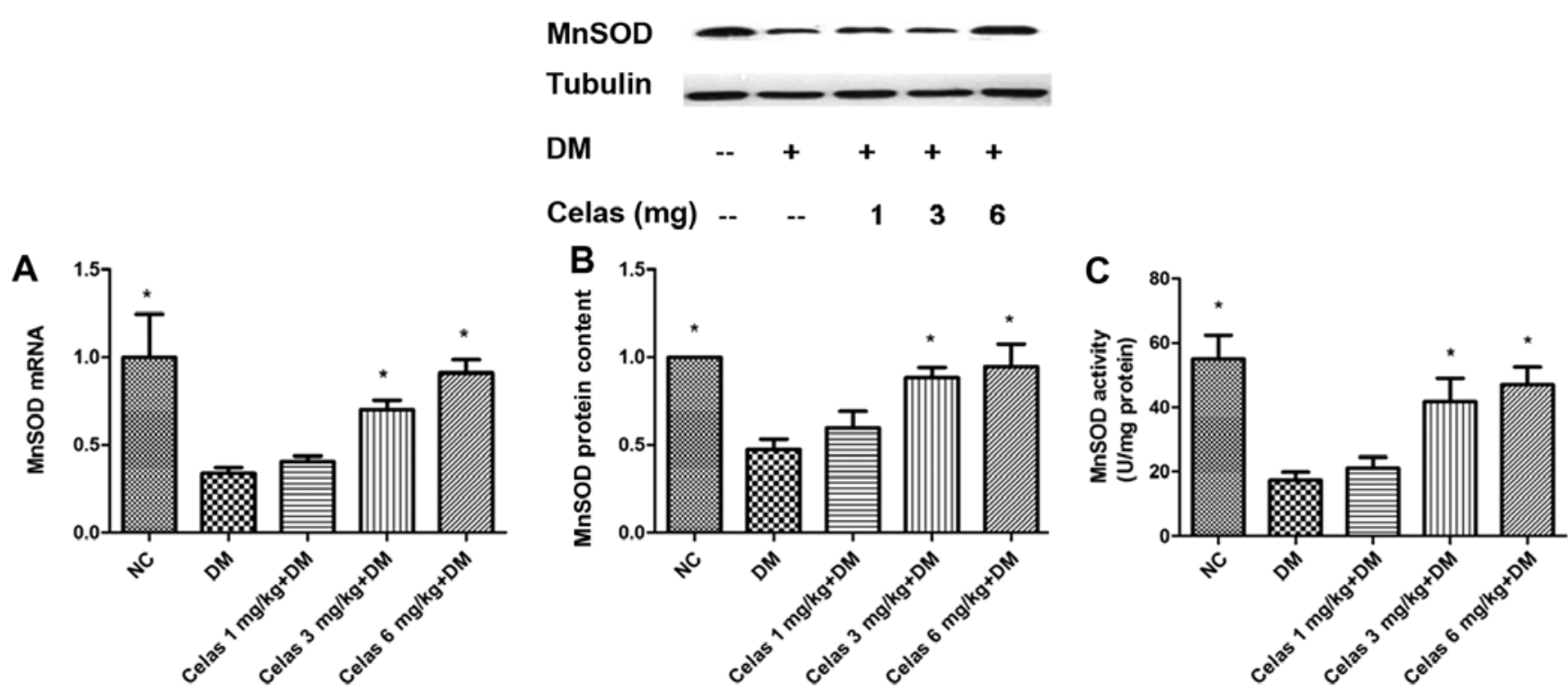

Figure 6. Celastrol attenuates the reduction in the expression and enzyme activity of mitochondrial manganese superoxide dismutase (MnSOD) in paravertebral muscle of rats. (A) Celastrol increased MnSOD mRNA levels in paravertebral muscle. (B) Celastrol increased MnSOD protein levels in paravertebral muscle. (C) Celastrol increased enzyme activity of MnSOD in paravertebral muscle. The activity of MnSOD was analyzed using relevant kits. "p $<0.05$ vs. diabetic rats.

the enzyme activity of MnSOD (Fig. 6C). However, neither the expression nor the enzyme activity of MnSOD exhibited a significant increase following treatment with $1 \mathrm{mg} / \mathrm{kg}$ celastrol. Taken together, these findings indicate that celastrol attenuates oxidative stress in a dose-dependent manner.

Celastrol promotes the activation of the AMPK-PGClaSIRT3 signaling pathway in skeletal muscle of rats with diabetes. To determine whether celastrol ameliorates oxidative stress by regulating SIRT3, we assessed the enzyme activity and content of SIRT3 in the paravertebral muscle of experiment rats. As expected, the mRNA and protein level of SIRT3 in the DM group was decreased by 68.4 and $65.3 \%$, respectively, compared to the NC group. Treatment with 3 and $6 \mathrm{mg} / \mathrm{kg}$ celastrol significantly increased both the mRNA and protein level of SIRT3 (Fig. 7A and D). Furthermore, we analyzed the enzyme activity of SIRT3. A reduction of $41.9 \%$ in SIRT3 enzyme activity was observed in the DM group compared with the NC group. Treatment with 3 and $6 \mathrm{mg} / \mathrm{kg}$ celastrol markedly enhanced the enzyme activity of SIRT3 (Fig. 7C). Consistent with the results obtained for MnSOD, neither the expression nor the enzyme activity of SIRT3 exhibited a marked improvement following treatment with $1 \mathrm{mg} / \mathrm{kg}$ celastrol.

It has been found that SIRT3 functions as a downstream target of PGC1 $\alpha$, which is directly regulated by AMPK $(14,15)$. Therefore, we examined the possible role of the AMPK-PGC1 $\alpha$ signaling pathway as a modulator of the regulatory effects of celastrol on SIRT3. The mRNA and protein levels of PGC1 $\alpha$ were decreased in the DM group by 66.3 and $64.9 \%$, respectively compared to the NC group (Fig. 7B and E). Treatment with 3 and $6 \mathrm{mg} / \mathrm{kg}$ celastrol significantly increased the levels PGC1 $\alpha$. In accordance with this upregulation, we also observed increased p-AMPK (Fig. 7F) levels in the paravertebral muscle of rats treated with 3 and $6 \mathrm{mg} / \mathrm{kg}$ celastrol. Similarly, there was no significant increase in the levels of PGC1 $\alpha$ and p-AMPK in the group treated with $1 \mathrm{mg} / \mathrm{kg}$ celastrol. Taken together, our results indicated that celastrol increased the expression 

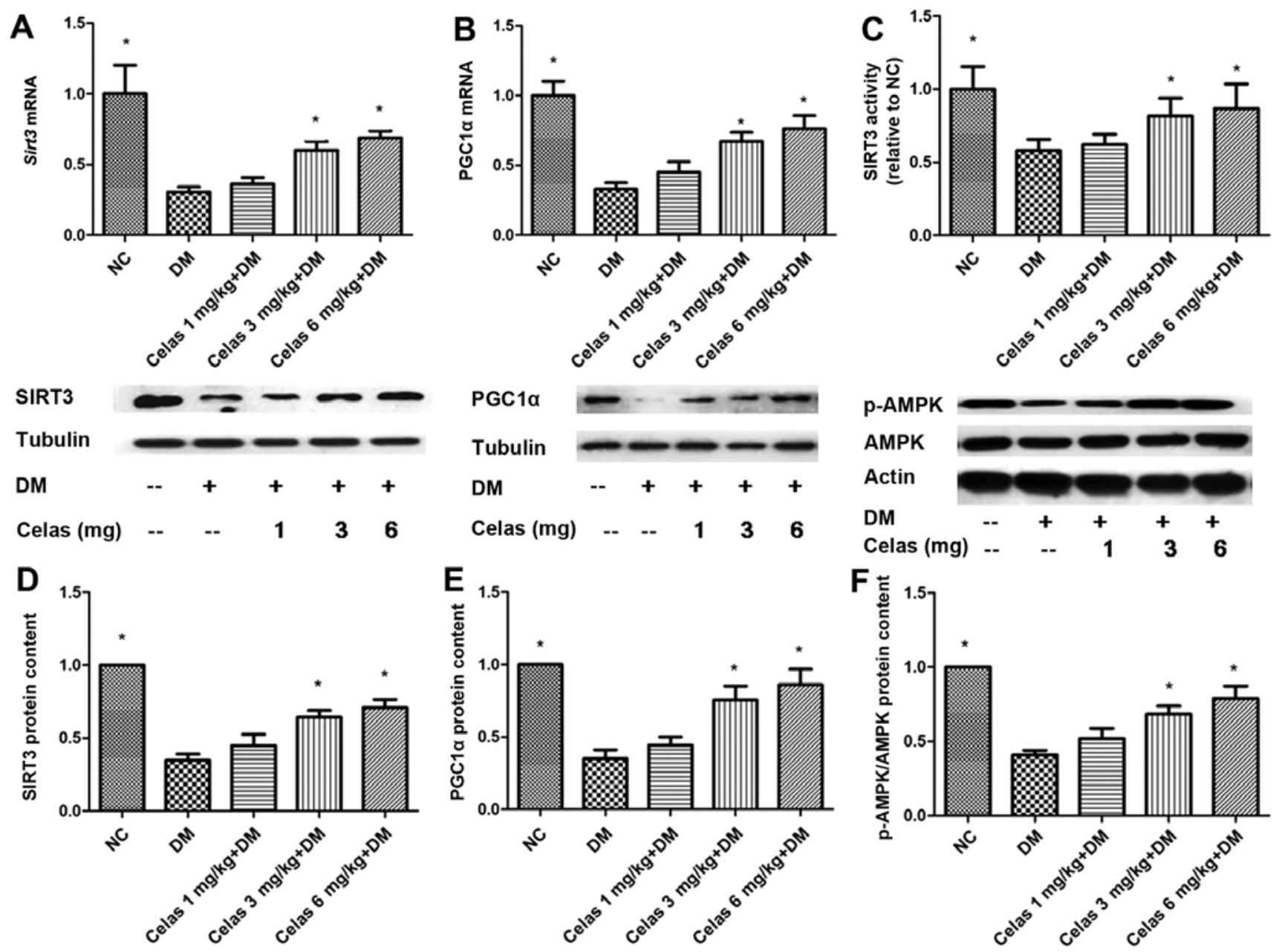

Figure 7. Celastrol upregulates the AMP-activated protein kinase (AMPK)-peroxisome proliferator-activated response- $\gamma$ coactivator $1 \alpha$ (PGC1 $\alpha$ )-silent matingtype information regulation 2 homolog 3 (SIRT3) signaling pathway in paravertebral muscle of rats. Celastrol increased (A) Sirt3 and (B) PGCl $\alpha$ mRNA levels in paravertebral muscle. (C) Celastrol increased SIRT3 activity in paravertebral muscle. Celastrol increased (D) SIRT3 and (E) PGC1 $\alpha$ protein levels in paravertebral muscle. (F) AMPK T-172 phosphorylation and AMPK total protein in the paravertebral muscle were assayed; AMPK phosphorylation was determined as p-AMPK normalized to total AMPK. Celastrol increased AMPK phosphorylation in muscle. "p $<0.05$ vs. diabetic rats.

of p-AMPK and PGC1 $\alpha$ in a dose-dependent manner in the skeletal muscle of rats with diabetes. Celastrol activated the AMPK-PGCl $\alpha$ signaling pathway in vivo.

\section{Discussion}

In this study, we firstly found that the expression levels of AMPK, PGC1 $\alpha$, SIRT3 and MnSOD were all decreased in the skeletal muscle of diabetic patients, accompanied by pathological damage. We also demonstrated that celastrol attenuated the pathological damage and oxidative stress, and activated the AMPK-PGC1 $\alpha$-SIRT3 signaling pathway in the skeletal muscle of diabetic rats.

Recently, ROS have been shown to play important roles in the activation of different signaling pathways and in the development of T2DM (7). Antioxidants, such as MnSOD, catalase (CAT), glutathione peroxidase (GPX) and GSH effectively form a defensive mechanism against the onslaught of ROS, protecting cells from oxidative stress (32). As is known, MDA is the main product of lipid peroxidation and its concentration usually reflects the total level of lipid peroxidation (33). In this study, both the activity and expression of MnSOD, as well as the level of GSH markedly decreased, with a significant increase in the level of MDA in diabetic rats, indicating that oxidative stress was enhanced during the development of diabetes. Following treatment with the middle and high dose ( 3 and $6 \mathrm{mg} / \mathrm{kg} / \mathrm{day}$ ) of celastrol, the activity and the expression of MnSOD, coupled with the level of GSH and MDA, returned towards their normal control values. Therefore it can be proposed that the middle and high dose of celastrol may be able to counteract oxidative stress-induced toxicity, whereas the low dose of celastrol (1 mg/ $\mathrm{kg} /$ day) did not have such an effect.

Furthermore, we explored the potential mechanisms of the celastrol-induced ameliorative effects related to oxidative stress in this study. SIRT3 is a mitochondrial sirtuin and regulates energy homeostasis and oxidative metabolism $(34,35)$. SIRT3 suppresses the cellular production of deleterious ROS, via deacetylation and the activation of MnSOD (36) and isocitrate dehydrogenase 2 (IDH2) $(37,38)$. Other studies have shown links between SIRT3 and mitochondrial ROS production by targeting HIF-1 $\alpha$ and SOD2 under different pathological and physiological conditions $(38,39)$. Recently, a study verified that the decreased level of SIRT3 in skeletal muscle in states of diabetes is a major component of the pathogenesis of T2DM, 
which can lead to altered mitochondrial function, increased ROS production and oxidative stress, and finally results in insulin resistance; SIRT3 expression in skeletal muscle is altered in models of both type 1 and 2 diabetes (40). In the present study, we found that SIRT3 expression was decreased in the skeletal muscle of patients and rats with T2DM. Following treatment with the middle and high dose of celastrol, SIRT3 expression was increased in the skeletal muscle of diabetic rats in accordance with the results obtained for MnSOD.

In this study, we also investigated the mechanism of celastrol-induced SIRT3 activation. It has been demonstrated that PGC1 $\alpha$, a nuclear transcriptional coactivator, increases SIRT3 expression at the mRNA and protein level (17). A previous study also reported that $\mathrm{PGC} 1 \alpha$ improved mouse SIRT3 activity in both hepatocytes and muscle cells, indicating that PGC1 $\alpha$ acts as an endogenous regulator of SIRT3 (41). The normal functioning of the PGC1 $\alpha /$ SIRT3 axis has been reported as essential for the regulation of mitochondrial metabolism, biogenesis and oxidative stress (12). Abnormal PGC1 $\alpha$ levels have been linked to the development of DM (42). In addition, AMPK increases the activity of PGC1 $\alpha$ (43), at least through two mechanisms. Firstly, PGC1 $\alpha$ is phosphorylated and activated by AMPK directly, which can then coactivate at its own promoter to stimulate its expression $(15,44)$. Next, AMPK increases the levels of cellular NAD ${ }^{+}$, in turn activating SIRT1 to activate PGC1 $\alpha(45,46)$. What is more, in this study, we found that the expression of AMPK and PGC1 $\alpha$ was decreased in the skeletal muscle of patients with T2DM. We presumed that celastrol upregulated SIRT3 expression via the activation of the AMPK-PGC1 $\alpha$ axis. As expected, our results revealed that the expression levels of AMPK and PGC1 $\alpha$ increased following treatment with the middle and high dose of celastrol. Thus, it can be concluded that celastrol regulates SIRT3 expression at least partly through the activation of the AMPK-PGC1 $\alpha$ axis.

This study has some limitations. First, this study did not employ special gene deficient mice, such as Sirt3 knockout mice, in order to provide more conclusive evidence of the signaling pathway in question. Second, cell culture was not conducted to verify the potential involvement of the AMPK-PGC1 $\alpha$-SIRT3 signaling pathway following treatment with celastrol in vitro.

In conclusion, the findings of the present study indicated that celastrol attenuated oxidative stress in skeletal muscle partial by regulating the AMPK-PGC1 $\alpha$-SIRT3 signaling pathway. Our findings suggest a potential role for celastrol in the treatment of the chronic complications of diabetes mellitus. Further studies on this matter are warranted using specific gene deficient mice and performing in vitro experiments to confirm our findings.

\section{References}

1. Andersen H, Schmitz O and Nielsen S: Decreased isometric muscle strength after acute hyperglycaemia in type 1 diabetic patients. Diabet Med 22: 1401-1407, 2005.

2. Andersen H, Gjerstad MD and Jakobsen J: Atrophy of foot muscles: A measure of diabetic neuropathy. Diabetes Care 27: 2382-2385, 2004.

3. Nyholm B, Qu Z, Kaal A, Pedersen SB, Gravholt CH, Andersen JL, Saltin B and Schmitz O: Evidence of an increased number of type IIb muscle fibers in insulin-resistant first-degree relatives of patients with NIDDM. Diabetes 46: 1822-1828, 1997.

4. Huang BK, Monu JU and Doumanian J: Diabetic myopathy: MRI patterns and current trends. AJR Am J Roentgenol 195: 198-204, 2010.
5. D'Souza DM, Al-Sajee D and Hawke TJ: Diabetic myopathy: Impact of diabetes mellitus on skeletal muscle progenitor cells. Front Physiol 4: 379, 2013.

6. Park SW, Goodpaster BH, Strotmeyer ES, de Rekeneire N, Harris TB, Schwartz AV, Tylavsky FA and Newman AB: Decreased muscle strength and quality in older adults with type 2 diabetes: The health, aging, and body composition study. Diabetes 55: 1813-1818, 2006.

7. Tangvarasittichai S: Oxidative stress, insulin resistance, dyslipidemia and type 2 diabetes mellitus. World J Diabetes 6: 456-480, 2015.

8. Tao R, Vassilopoulos A, Parisiadou L, Yan Y and Gius D: Regulation of MnSOD enzymatic activity by Sirt3 connects the mitochondrial acetylome signaling networks to aging and carcinogenesis. Antioxid Redox Signal 20: 1646-1654, 2014.

9. Montano MA, Barrio Lera JP, Gottlieb MG, Schwanke CH, da Rocha MI, Manica-Cattani MF, dos Santos GF and da Cruz IB: Association between manganese superoxide dismutase (MnSOD) gene polymorphism and elderly obesity. Mol Cell Biochem 328: 33-40, 2009.

10. Kahn BB, Alquier T, Carling D and Hardie DG: AMP-activated protein kinase: Ancient energy gauge provides clues to modern understanding of metabolism. Cell Metab 1: 15-25, 2005.

11. Kukidome D, Nishikawa T, Sonoda K, Imoto K, Fujisawa K, Yano M, Motoshima H, Taguchi T, Matsumura $\mathrm{T}$ and Araki E: Activation of AMP-activated protein kinase reduces hyperglycemia-induced mitochondrial reactive oxygen species production and promotes mitochondrial biogenesis in human umbilical vein endothelial cells. Diabetes 55: 120-127, 2006.

12. Bell EL and Guarente L: The SirT3 divining rod points to oxidative stress. Mol Cell 42: 561-568, 2011.

13. Schwer B, North BJ, Frye RA, Ott M and Verdin E: The human silent information regulator (Sir)2 homologue hSIRT3 is a mitochondrial nicotinamide adenine dinucleotide-dependent deacetylase. J Cell Biol 158: 647-657, 2002.

14. Kong X, Wang R, Xue Y, Liu X, Zhang H, Chen Y, Fang F and Chang Y: Sirtuin 3, a new target of PGC-1 $\alpha$, plays an important role in the suppression of ROS and mitochondrial biogenesis. PLoS One 5: el1707, 2010.

15. Jäger S, Handschin C, St-Pierre $J$ and Spiegelman BM: AMP-activated protein kinase (AMPK) action in skeletal muscle via direct phosphorylation of PGC-1alpha. Proc Natl Acad Sci USA 104: 12017-12022, 2007.

16. Wang Q, Li L, Li CY, Pei Z, Zhou M and Li N: SIRT3 protects cells from hypoxia via PGC-1 $\alpha$ - and MnSOD-dependent pathways. Neuroscience 286: 109-121, 2015.

17. Rato L, Duarte AI, Tomás GD, Santos MS, Moreira PI, Socorro S, Cavaco JE, Alves MG and Oliveira PF: Pre-diabetes alters testicular PGC1- $\alpha /$ SIRT3 axis modulating mitochondrial bioenergetics and oxidative stress. Biochim Biophys Acta 1837: 335-344, 2014.

18. Hao J, Hao C, Zhang L, Liu X, Zhou X, Dun Y, Li H, Li G, Zhao X, An Y, et al: OM2, a novel oligomannuronate-chromium(III) complex, promotes mitochondrial biogenesis and lipid metabolism in 3T3-L1 adipocytes via the AMPK-PGC1 $\alpha$ pathway. PLoS One 10: e0131930, 2015.

19. Gu L, Bai W, Li S, Zhang Y, Han Y, Gu Y, Meng G, Xie L, Wang J, Xiao Y, et al: Celastrol prevents atherosclerosis via inhibiting LOX-1 and oxidative stress. PLoS One 8: e65477, 2013.

20. Ge Y, Xie H, Li S, Jin B, Hou J, Zhang H, Shi M and Liu Z: Treatment of diabetic nephropathy with Tripterygium wilfordii Hook F extract: A prospective, randomized, controlled clinical trial. J Transl Med 11: 134, 2013.

21. Yu X, Tao W, Jiang F, Li C, Lin J and Liu C: Celastrol attenuates hypertension-induced inflammation and oxidative stress in vascular smooth muscle cells via induction of heme oxygenase-1. Am J Hypertens 23: 895-903, 2010.

22. Rato L, Alves MG, Dias TR, Lopes G, Cavaco JE, Socorro S and Oliveira PF: High-energy diets may induce a pre-diabetic state altering testicular glycolytic metabolic profile and male reproductive parameters. Andrology 1: 495-504, 2013.

23. Ai J, Wang N, Yang M, Du ZM, Zhang YC and Yang BF: Development of Wistar rat model of insulin resistance. World $\mathbf{J}$ Gastroenterol 11: 3675-3679, 2005

24. Frazier AE and Thorburn DR: Biochemical analyses of the electron transport chain complexes by spectrophotometry. Methods Mol Biol 837: 49-62, 2012.

25. Brandauer J, Vienberg SG, Andersen MA, Ringholm S, Risis S, Larsen PS, Kristensen JM, Frøsig C, Leick L, Fentz J, et al: AMP-activated protein kinase regulates nicotinamide phosphoribosyl transferase expression in skeletal muscle. J Physiol 591: 5207-5220, 2013 
26. Mikhed Y, Daiber A and Steven S: Mitochondrial oxidative stress, mitochondrial DNA damage and their role in age-related vascular dysfunction. Int J Mol Sci 16: 15918-15953, 2015.

27. Brandauer J, Andersen MA, Kellezi H, Risis S, Frøsig C, Vienberg SG and Treebak JT: AMP-activated protein kinase controls exercise training- and AICAR-induced increases in SIRT3 and MnSOD. Front Physiol 6: 85, 2015.

28. Kim JE, Lee MH, Nam DH, Song HK, Kang YS, Lee JE, Kim HW, Cha JJ, Hyun YY, Han SY, et al: Celastrol, an NF- $\mathrm{B}$ inhibitor, improves insulin resistance and attenuates renal injury in $\mathrm{db} / \mathrm{db}$ mice. PLoS One 8: e62068, 2013.

29. Lodovici M, Bigagli E, Luceri C, Mannucci E, Rotella CM and Raimondi L: Gender-related drug effect on several markers of oxidation stress in diabetes patients with and without complications. Eur J Pharmacol 766: 86-90, 2015.

30. Feng B, Yan XF, Xue JL, Xu L and Wang H: The protective effects of $\alpha$-lipoic acid on kidneys in type 2 diabetic Goto-Kakisaki rats via reducing oxidative stress. Int J Mol Sci 14: 6746-6756, 2013.

31. Lawler JM, Kwak HB, Kim JH and Suk MH: Exercise training inducibility of MnSOD protein expression and activity is retained while reducing prooxidant signaling in the heart of senescent rats. Am J Physiol Regul Integr Comp Physiol 296 : R1496-R1502, 2009.

32. Srinivasan M, Rajendra Prasad N and Menon VP: Protective effect of curcumin on gamma-radiation induced DNA damage and lipid peroxidation in cultured human lymphocytes. Mutat Res 611: 96-103, 2006.

33. Ayala A, Muñoz MF and Argüelles S: Lipid peroxidation: Production, metabolism, and signaling mechanisms of malondialdehyde and 4-hydroxy-2-nonenal. Oxid Med Cell Longev 2014 360438, 2014.

34. Onyango P, Celic I, McCaffery JM, Boeke JD and Feinberg AP: SIRT3, a human SIR2 homologue, is an NAD-dependent deacetylase localized to mitochondria. Proc Natl Acad Sci USA 99: 13653-13658, 2002.

35. Schwer B, North BJ, Frye RA, Ott M and Verdin E: The human silent information regulator (Sir) 2 homologue hSIRT3 is a mitochondrial nicotinamide adenine dinucleotide-dependent deacetylase. J Cell Biol 158: 647-657, 2002.

36. Tao R, Coleman MC, Pennington JD, Ozden O, Park SH, Jiang H, Kim HS, Flynn CR, Hill S, Hayes McDonald W, et al: Sirt3-mediated deacetylation of evolutionarily conserved lysine 122 regulates MnSOD activity in response to stress. Mol Cell 40: 893-904, 2010.
37. Qiu X, Brown K, Hirschey MD, Verdin E and Chen D: Calorie restriction reduces oxidative stress by SIRT3-mediated SOD2 activation. Cell Metab 12: 662-667, 2010.

38. Someya S, Yu W, Hallows WC, Xu J, Vann JM, Leeuwenburgh C, Tanokura M, Denu JM and Prolla TA: Sirt3 mediates reduction of oxidative damage and prevention of age-related hearing loss under caloric restriction. Cell 143: 802-812, 2010.

39. Bell EL, Emerling BM, Ricoult SJ and Guarente L: SirT3 suppresses hypoxia inducible factor $1 \alpha$ and tumor growth by inhibiting mitochondrial ROS production. Oncogene 30: 2986-2996, 2011.

40. Jing E, Emanuelli B, Hirschey MD, Boucher J, Lee KY, Lombard D, Verdin EM and Kahn CR: Sirtuin-3 (Sirt3) regulates skeletal muscle metabolism and insulin signaling via altered mitochondrial oxidation and reactive oxygen species production. Proc Natl Acad Sci USA 108: 14608-14613, 2011.

41. Park SJ, Ahmad F, Philp A, Baar K, Williams T, Luo H, Ke H, Rehmann H, Taussig R, Brown AL, et al: Resveratrol ameliorates aging-related metabolic phenotypes by inhibiting cAMP phosphodiesterases. Cell 148: 421-433, 2012.

42. Joseph AM, Joanisse DR, Baillot RG, Hood DA: Mitochondrial dysregulation in the pathogenesis of diabetes: potential for mitochondrial biogenesis-mediated interventions. Exp Diabetes Res 2012: 642038, 2012.

43. Lin J, Handschin C and Spiegelman BM: Metabolic control through the PGC-1 family of transcription coactivators. Cell Metab 1: 361-370, 2005.

44. Handschin C, Rhee J, Lin J, Tarr PT and Spiegelman BM: An autoregulatory loop controls peroxisome proliferator-activated receptor gamma coactivator lalpha expression in muscle. Proc Natl Acad Sci USA 100: 7111-7116, 2003.

45. Cantó C, Gerhart-Hines Z, Feige JN, Lagouge M, Noriega L, Milne JC, Elliott PJ, Puigserver P and Auwerx J: AMPK regulates energy expenditure by modulating $\mathrm{NAD}^{+}$metabolism and SIRT1 activity. Nature 458: 1056-1060, 2009.

46. Fulco M, Cen Y, Zhao P, Hoffman EP, McBurney MW, Sauve AA and Sartorelli V: Glucose restriction inhibits skeletal myoblast differentiation by activating SIRT1 through AMPK-mediated regulation of Nampt. Dev Cell 14: 661-673, 2008. 